\title{
Calibration Function for the Orbitrap FTMS Accounting for the Space Charge Effect
}

\author{
Mikhail V. Gorshkov, ${ }^{a}$ David M. Good, ${ }^{\text {b }}$ Yaroslav Lyutvinskiy, ${ }^{\text {b }}$ \\ Hongqian Yang, ${ }^{\mathrm{b}}$ and Roman A. Zubarev ${ }^{\mathrm{b}}$ \\ ${ }^{a}$ Institute for Energy Problems of Chemical Physics, Moscow, Russia \\ ${ }^{\mathrm{b}}$ Karolinska Institute, Stockholm, Sweden
}

\begin{abstract}
Ion storage in an electrostatic trap has been implemented with the introduction of the Orbitrap Fourier transform mass spectrometer (FTMS), which demonstrates performance similar to high-field ion cyclotron resonance MS. High mass spectral characteristics resulted in rapid acceptance of the Orbitrap FTMS for Life Sciences applications. The basics of Orbitrap operation are well documented; however, like in any ion trap MS technology, its performance is limited by interactions between the ion clouds. These interactions result in ion cloud couplings, systematic errors in measured masses, interference between ion clouds of different size yet with close $\mathrm{m} / \mathrm{z}$ ratios, etc. In this work, we have characterized the space-charge effect on the measured frequency for the Orbitrap FTMS, looking for the possibility to achieve sub-ppm levels of mass measurement accuracy (MMA) for peptides in a wide range of total ion population. As a result of this characterization, we proposed an $\mathrm{m} / \mathrm{z}$ calibration law for the Orbitrap FTMS that accounts for the total ion population present in the trap during a data acquisition event. Using this law, we were able to achieve a zero-space charge MMA limit of $80 \mathrm{ppb}$ for the commercial Orbitrap FTMS system and sub-ppm level of MMA over a wide range of total ion populations with the automatic gain control values varying from 10 to 107. (J Am Soc Mass Spectrom 2010, 21, 1846-1851) (C) 2010 American Society for Mass Spectrometry
\end{abstract}

$\mathrm{T}$ wo major approaches, known as "bottom-up" and "top-down", are used for identification of peptides by mass spectrometry [1-4]. Both approaches benefit from high mass accuracy [5-7]. The number of possible sequence candidates decreases rapidly with increasing molecular mass accuracy (MMA). The MMA of $100 \mathrm{ppb}$ (the level yet to be achieved) is in general insufficient for unique identification of a peptide by its molecular mass, although it allows assigning a peptidogenic elemental composition for precursor peptide ions up to a mass of $1000 \mathrm{Da}$ [8-10]. However, the $1 \mathrm{ppm}$ mass accuracy, which is routinely available today [11] in Fourier transform ion cyclotron resonance mass spectrometry (FTICR MS), is often sufficient for deducing unique amino acid composition (excluding the case of the isomeric leucine and isoleucine residues) for an arbitrary peptide below ca. $600 \mathrm{Da}$ [8]. Also, as has been suggested recently, some peptides do possess molecular masses that are unique within the genome [12]. When measured with sufficiently high accuracy, such masses can be used as "accurate mass tags" for unambiguous protein identification [13]. The required

Address reprint requests to Professor R. A. Zubarev, Division of Molecular Biometry, Department of Medicinal Biochemistry and Biophysics, Karolinska Institutet, Scheelesväg 2, SE 17177 Stockholm, Sweden. E-mail: roman.zubarev@ki.se or Dr. Mikhail V. Gorshkov, Institute for Energy Problems of Chemical Physics, Russian Academy of Sciences, 38 Leninsky Pr., Bld.2, 119334 Moscow, Russia. E-mail: gorshkov@chph.ras.ru mass accuracy is below $10 \mathrm{ppm}$ for the peptides with molecular weights above $2500 \mathrm{Da}$ and below $1 \mathrm{ppm}$ level for $1 \mathrm{kDa}$ peptides.

In high-performance mass spectrometers, it is usually easier to obtain high-resolution than high mass accuracy. For instance, FTICR MS can resolve ions of $>1 \mathrm{kDa}$ polypeptides differing by less than the mass of an electron $(0.545 \mathrm{mDa})$ [8]. The demonstrated resolving power of $>3000,000$ should be sufficient for routine mass accuracy of $100 \mathrm{ppb}$, yet the $1 \mathrm{ppm}$ "barrier" for peptide masses turned out to be surprisingly resilient in FT ICR. The progress in MS technology in recent years created a line of commercially available products with previously unimaginable performance. The most notable example is the recently introduced 3D electrostatic FT mass-analyzer Orbitrap [14]. This instrument provides resolving power exceeding that of modern time-of-flight MS and mass measurement accuracy comparable with that of FTICR MS. For example, MMA of less than 1 ppm over a dynamic range of 5000 (max/min intensity ratios) has been reported using the commercial hybrid LTQ Orbitrap FTMS system [15, 16].

The Orbitrap's operation is based upon the ion orbital trapping in an electrostatic field with a 3D potential distribution described elsewhere in cylindrical coordinates $(r, Z)$ as follows [14]: 


$$
\varphi(r, Z)=\frac{k}{2}\left(Z^{2}-\frac{r^{2}}{2}\right)+\frac{k}{2} R_{m}^{2} \ln \left(\frac{r}{R_{m}}\right)+C
$$

in which $k$ is the field curvature, $C$ is a constant, and $R_{m}$ is the characteristic radius. The ion trajectory in such a field exhibits a combination of three decoupled fundamental motions: a rotation around the central electrode, radial oscillations around the middle trajectory of rotation, and axial oscillations along the central electrode. Because the potential distribution along the Z-axis is parabolic, the frequency of the latter oscillations is independent of their amplitude. These axial oscillations are recorded as time domain image current signals followed by Fast Fourier transform (FFT) to extract the frequencies. To derive an analytical expression for the frequency of axial oscillations we may assume that ion's motion in radial plane and along the Z-axis are completely decoupled and consider the general equation of ion motion as:

$$
m \frac{d^{2} Z}{d t^{2}}=q E_{z}
$$

in which $E_{z}$ is defined by the potential $\varphi(r, Z)$ as follows: $E_{z}=-\partial \varphi / d Z$. From eq 1 , the latter results in the following equation of motion (assuming that $C, k$, and $R_{m}$ are independent of Z):

$$
m \frac{d^{2} Z}{d t^{2}}=-q \frac{\partial \varphi}{d Z}=-q k Z
$$

Assuming that Z-motion of ions in an Orbitrap is periodic with the frequency $f$, eq 3 presents the common equation of motion for harmonic oscillator, $Z^{\prime \prime}+(2 \pi f)^{2} Z=$ 0 . Therefore, one can easily obtain the general equation for the measured frequency, $f$, in an Orbitrap:

$$
f=\frac{1}{2 \pi} \sqrt{\frac{k}{(m / z)}}
$$

in which $\mathrm{m} / \mathrm{z}$ is the mass-to-charge ratio of the ions.

Eq 4 gives the following "basic" calibration equation for the Orbitrap FTMS:

$$
\frac{m}{z}=\frac{A}{f^{2}}
$$

where $A$ is the calibration constant. Note, that the field curvature $k$ in eq 1 is proportional to the amplitude of the electrostatic voltage, $V_{c}$, applied to the central electrode, and, therefore, from eq $4, f \sim \sqrt{V_{c}}$. Eq 4 is similar to the equation for ions' axial oscillations in an FTICR trap in harmonic extension of the corresponding trapping electrostatic field [17]. In other words, the measurement of the axial oscillations in FTICR MS is a proper analogy to that in an Orbitrap FTMS. In both cases, the measured frequency depends on the amplitude of electrostatic field, trap geometry, and the $\mathrm{m} / \mathrm{z}$ ratio. However, application of the Orbitrap's principle of detection based on the measurements of ions' axial oscillations to FTICR MS presents a challenge. Indeed, to increase the frequency of axial oscillations in FTICR up to the comparable values (from the resolving power viewpoint), one has to increase the amplitude of electrostatic voltage applied to the FTICR trap electrodes. For example, for a 5-cm cubic FTICR trap with a perfectly harmonic trapping field, the axial oscillation frequency of, e.g., 100,000 $\mathrm{Hz}$ for the $m / z 1000$ will be achieved at the voltage of $\sim 2000 \mathrm{~V}_{\mathrm{DC}}$. However, at this voltage the upper limit of $m / z$ being trapped in the cyclotron motion plane of the FTICR trap (critical mass) will be $\sim 1000$ for a magnetic field of 10 Tesla. In reality, the actual critical $m / z$, at which the ions are not trapped in the cyclotron plane, may be even less [18]. This makes implementation of the Orbitrap's signal measurement principles in FTICR MS impractical. Moreover, while the harmonization of ions' axial oscillations may be possible over the whole volume of the FTICR trap [19], the axial frequency shifts due to the magnetic field inhomogeneity [20] will result in unacceptable systematic errors in the measured masses.

Space-charge from the ions present in the Orbitrap changes the effective trapping field. These changes can be considered as small perturbations in the electrostatic field that affect the measured frequency of ions' axial oscillations. In general, the measured frequency becomes dependent on the total space charge generated by the ions, which is typically controlled by the automatic gain control (AGC). Therefore, studying the effect of changing AGC value reveals the functional dependence of the ions' behavior on the total space charge. In this work we were interested in the effect of the AGC value on the measured frequencies of ions' oscillations. As it was well documented in the FTICR MS literature, there are several models developed for FTICR MS ion traps of different geometries to account for the spacecharge-dependent shifts in measured frequency [21-25]. According to these models, the space-charge shift in the measured cyclotron frequency is linearly proportional to the number of ions. This property of the FTICR MS was reflected in a number of calibration functions used to take into account the amount of trapped ions [22, 26-30]. The purpose of this work was to experimentally determine the functional dependence of the measured frequency of ions' axial oscillations in an Orbitrap FTMS on the total number of ions and develop the calibration equation that takes this into account.

\section{Experimental}

In this work, we used a commercial LTQ Orbitrap Velos mass spectrometer (ThermoScientific, Bremen, Germany) to study the mass accuracy and resolving power effects in an Orbitrap. The instrument was calibrated using an automatic routine based on a standard calibra- 


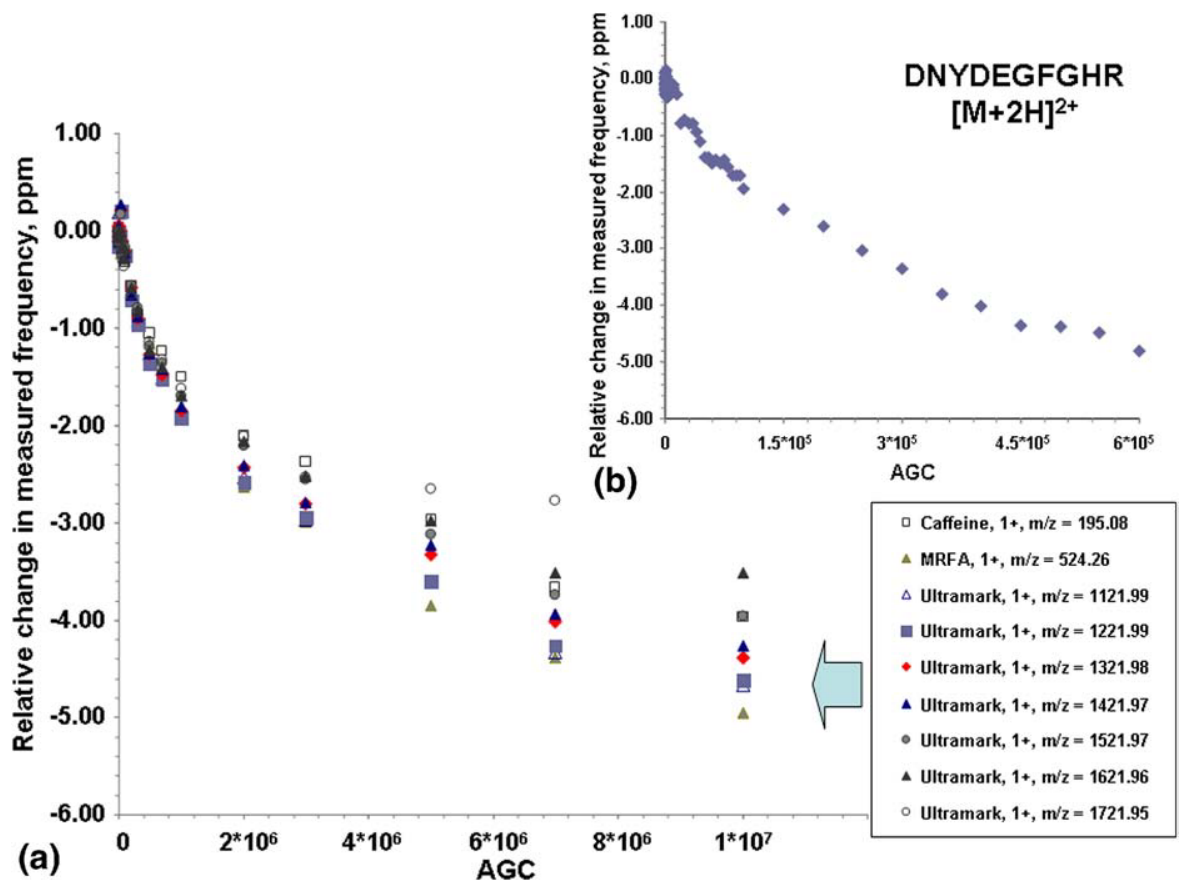

(a)

Figure 1. Dependence of the relative change in the measured frequency on pre-set AGC value for a variety of ions from the MRFA-Ultramark 1621 calibration mixture (a) and the separately loaded doubly-charged synthetic peptide DNYDEGFGHR (b).

tion solution containing caffeine, peptide MRFA, and Ultramark 1621 (Sigma Chemical Company, St. Louis, USA). This external mass scale calibration was used only for peak identification purposes, as all mass spectra were then internally calibrated using several known ions. The solutions were electrosprayed using the Proxeon (Odense, Denmark) nano-ESI interface through a metal-coated nano-ESI pulled glass capillary (Protana Engineering, Odense, Denmark) at a flow rate of $200 \mathrm{~nL} / \mathrm{min}$. The voltage applied to the capillary was $+1 \mathrm{kV}$, and the desolvation heated metal capillary of the LTQ was maintained at $140{ }^{\circ} \mathrm{C}$. Xcalibur 2.1 software was used for control of the experimental event sequence as well as visualization, processing, and storage of mass spectral data. The transient time domain signals obtained by broad-band differential dipolar detection were amplified and digitized followed by apodization and magnitude-mode Fast Fourier transform. Frequency measurements were based on the centroids of the monoisotopic peaks. Calculations of exact masses of peptides were made using the latest AME2003 atomic mass evaluation [31].

\section{Results}

The LTQ Orbitrap instrument possesses the AGC functionality, which allows one to accumulate in the trap the same number of ions each time, according to the preset AGC value. We assume here that AGC is proportional to the total number of ions loaded into the trap. This assumption may not be correct in the case of nonuniform ion beams, pulsed ionization sources, or due to instabilities in the source's operation. Therefore, in the further analysis we consider all studied effects as dependent on the instrument-provided AGC value rather than the real physical parameter such as the actual number of ions present in the trap. Figure 1a shows dependence of the frequencies measured for the ions from the MRFA-Ultramark 1621 calibration mixture. Similar dependence was observed for synthetic peptides (shown in Figure 1b) in separate experiments. However, the rate of the frequency decrease for doubly charged peptide ions was two times higher than for the singly charge ions of similar $\mathrm{m} / \mathrm{z}$ ratio from the MRFAUltramark 1621 mixture. This contradicts the general assumption that the frequency shift due to the space charge effect has to be $m / z$-dependent, and independent

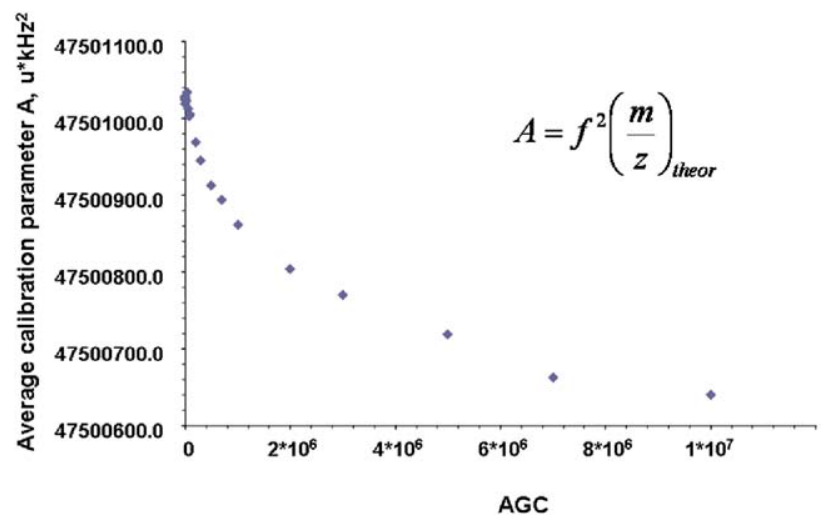

Figure 2. Dependence of the calibration constant $A$ defined by eq 6 and averaged for all the ions studied on AGC value. 


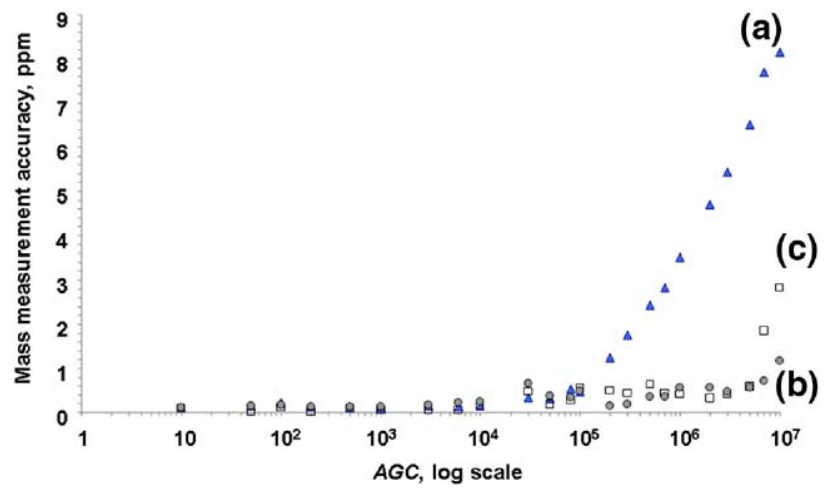

Figure 3. Dependence of the mass measurement accuracy (MMA) obtained for the ions from MRFA-Ultramark 1621 calibration mixture using different calibration functions on AGC value: (a) zero-space charge approximation (eq 5); (b) $\sqrt{A G C}$-dependent calibration function found from this study (eq 7); and (c) instrument's built-in calibration function (eq 9) with calibration constants empirically determined for different ranges of AGC.

of the charge state. We do not have an explanation for this observation, and it thus warrants further investigation using ions with different $\mathrm{m} / \mathrm{z}$ ratios, selectively isolated in the LTQ before injection into the C-trap. However, our preliminary studies on the relative frequency shift with AGC using multiply charged cytochrome $c$ ions (results not shown) supported the results from MRFA-Ultramark 1621 experiments shown in Figure 1a. Note that the frequency was shifting downward with increasing AGC value, following the function of $\sqrt{A G C}$. This demonstrates a difference between the frequencies measured in Orbitrap FTMS and FTICR MS. In the latter, the frequency shifts downward linearly with AGC. The reason for the differences in the frequency behavior may be due to limitations implied for the Orbitrap by the presence of C-trap with limited space charge capacity. However, some reports suggest that the ejection part of the LTQ has lesser space charge capacity compared with the C-trap [32] and, thus, the limitation of the number of charges loaded into the Orbitrap analyzer may be related to the other elements of the ion guide. The uncertainties with this issue warrant further studies.

The measured frequency was further used to calculate calibration coefficient $A$ in eq 3 . For each of the ions from the calibration mixture, this coefficient was determined as

$$
A_{i}=f_{i}^{2}\left(\frac{m}{z}\right)_{i, \text { theor }}
$$

followed by the averaging of $A_{i}$ values for all ions measured at the particular AGC value. Figure 2 shows dependence of the average calibration coefficient on the AGC. Expectedly, it also depends on $\sqrt{A G C}$. Given the result shown in Figure 2 and eq 5, we can write the following calibration equation for the Orbitrap FTMS that takes into account the total ion population in the trap:

$$
\frac{m}{z}=\frac{A}{f^{2}}+\frac{B \sqrt{A G C}}{f^{2}}
$$

in which $A$ is the zero-space charge term, and $B$ is the second coefficient determined by the dependence of the measured frequency on AGC. Both terms in the calibration equation can be determined empirically. The best fit of dependence shown in Figure 2 using the equation $A+B \sqrt{A G C}$ resulted in the following coefficients in eq 7:

$$
\begin{aligned}
& A=47501027.5(3.8) \mathrm{u} *(\mathrm{kHz})^{2} ; \\
& B=-0.13674(39) \mathrm{u} *(\mathrm{kHz})^{2}
\end{aligned}
$$

with $R^{2}=0.993$ for the fit. Note that these coefficients are specific for the trap size and the amplitude of the trapping voltage applied to the central electrode. When these parameters change the $\mathrm{m} / \mathrm{z}$ scale has to be recalibrated. The calibration equation coefficients can also be specific to experimental parameters. For example,

\begin{tabular}{|c|c|c|c|c|c|}
\hline \multirow[b]{2}{*}{ Substance } & \multirow[b]{2}{*}{ Theoretical $\mathrm{m} / \mathrm{z}$} & \multicolumn{2}{|c|}{ AGC range: 10 to $10^{6}$} & \multicolumn{2}{|c|}{ AGC range: 10 to $10^{7}$} \\
\hline & & $\begin{array}{c}\text { Average } \\
\text { measured } \mathrm{m} / \mathrm{z}\end{array}$ & $\begin{array}{l}\text { Relative mass } \\
\text { deviation, ppb }\end{array}$ & $\begin{array}{c}\text { Average } \\
\text { measured } \mathrm{m} / \mathrm{z}\end{array}$ & $\begin{array}{l}\text { Relative mass } \\
\text { deviation, ppb }\end{array}$ \\
\hline Caffeine & 195.087652 & 195.0876581 & 31 & 195.087643 & -44 \\
\hline MRFA-acetate & 524.264964 & 524.264820 & -193 & 524.264921 & -82 \\
\hline Utmk1621 & 1121.997024 & 1121.99700 & -24 & 1121.997202 & 159 \\
\hline Utmk1621 & 1221.990636 & 1221.99053 & -86 & 1221.990725 & 73 \\
\hline Utmk1621 & 1321.984249 & 1321.98411 & -107 & 1321.984220 & -21 \\
\hline Utmk1621 & 1421.977862 & 1421.97791 & 34 & 1421.977992 & 92 \\
\hline Utmk1621 & 1521.971475 & 1521.97134 & -89 & 1521.971301 & -114 \\
\hline Utmk1621 & 1621.965088 & 1621.96464 & -274 & 1621.964448 & -395 \\
\hline Utmk1621 & 1721.958701 & 1721.95884 & 81 & 1721.958279 & -245 \\
\hline \multicolumn{3}{|c|}{ Average MMA across the whole $\mathrm{m} / \mathrm{z}$ region } & 102 & & 136 \\
\hline
\end{tabular}
vacuum conditions in the C-trap region may affect its

Table 1. Self-consistency test of the Orbitrap calibration using eqs 7 and 8 . The relative deviations between measured and theoretical masses have been calculated for two AGC regions. Self-consistency test of MMA for MRFA-Ultramark 1621 calibration mixture 


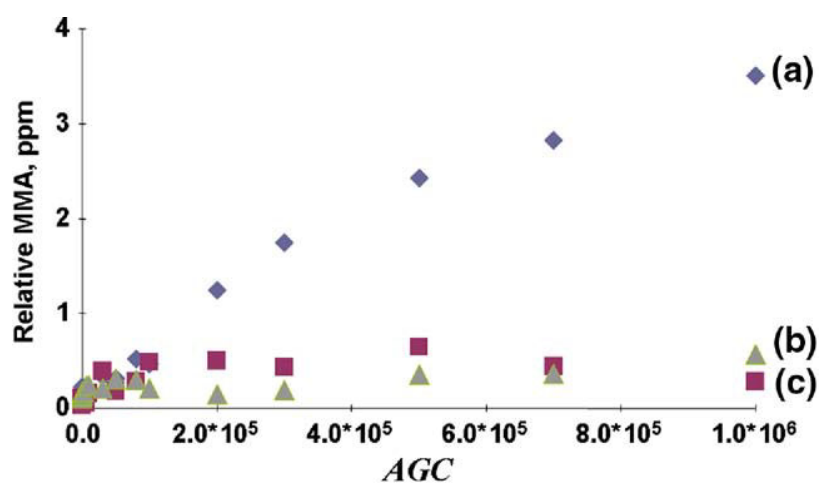

Figure 4. Dependence of the relative mass measurement accuracy obtained for the ions from MRFA-Ultramark 1621 calibration mixture using calibration function proposed in this work (eqs 7 and 8 ) in the low AGC range from 10 to $10^{6}$.

space charge capacity and, thus, the frequency dependence on AGC. The error in first term $A$ represents the ultimate error in mass determination for the Orbitrap FTMS instrument used in this work in zero-space charge approximation. From eq 8 , this error was $80 \mathrm{ppb}$ for our measurements. Figure 3 shows comparison of the MMA for the calibration mixture MRFA-Ultramark 1621 used in this study and calculated using both the basic Orbitrap's calibration eq 5 and the space-charge dependent eq 7 over the range of AGC values from 10 to $10^{7}$. Note, that the LTQ Orbitrap Velos also utilizes the AGC-dependent calibration function, which can reportedly be written as follows:

$$
\frac{m}{z}=\frac{A}{f^{2}}+\frac{B}{f^{3}}
$$

in which both parameters have different values and functional dependences for three different ranges of AGCs. The results of the MMA obtained for the same mixture using built-in calibration function are also shown in Figure 3. We believe that the calibration eq 5 presents a better opportunity to achieve below $100 \mathrm{ppb}$ mass measurement accuracy as it reflects the fundamental properties of the Orbitrap's operation rather than an empirical fit by the regression function. Table 1 summarizes the results of calibration using MRFAUltramark 1621 mixture. The calibration constant from eq 8 were used to calculate average $\mathrm{m} / \mathrm{z}$ ratios for the mixture substances in two AGC regions: $10-10^{6}$ and $10-10^{7}$. At low AGC values as shown in Figure 4, we have received relative mass deviation error of $100 \mathrm{ppb}$ that supports the zero-space charge MMA limit for the Orbitrap instrument used in this work.

\section{Conclusion}

The functional dependence of the measured frequency on AGC allows obtaining high MMA in a wider range of the number of trapped ions. Contrary to the FTICR MS, the measured frequency in Orbitrap depends on the square root of the total number of trapped ions. Based on this observation, we introduced a calibration equation for the Orbitrap. Using this equation, we were able to obtain a MMA of $\sim 100 \mathrm{ppb}$ over the $\mathrm{m} / \mathrm{z}$ range from 100 to 2000 for AGCs varying from 10 to 10,000, and sub-ppm MMA for up to $10^{7}$ AGC values.

\section{Acknowledgments}

The authors acknowledge support for this work by grant VR-NT, 2007-4410. M.V.G. also thanks the Russian Basic Science Foundation (grants 08-04-91,121 and 08-04-01,339) and the U.S. Civilian Research and Development Foundation (grant RUB1-2909-MO07). D.M.G. is grateful for a post-doctoral fellowship from the Wenner-Gren Foundation.

\section{References}

1. Mann, M.; Hojrup, P.; Roepstorff, P. Use of Mass Spectrometric Molecular Weight Information to Identify Proteins in Sequence Database. Biol. Mass Spectrom. 1993, 22, 338-345.

2. Shevchenko, A.; Jensen, O. N.; Podtelejnikov, A. V.; Sagliiocco, F.; Wilm, M.; Vorm, O.; Mortensen, P.; Shevchenko, A.; Boucherie, H.; Mann, M. Linking Genome and Proteome by Mass Spectrometry: Large-Scale Identification of Yeast Proteins From Two-Dimensional Gels. Proc. Natl. Acad. Sci. U.S.A. 1996, 93, 14440-14445.

3. Yates, J. R. III. Mass Spectrometry: From Genomics to Proteomics. Trends Genet. 2000, 16, 5-8.

4. Kelleher, N. L.; Lin, H. Y.; Valaskovic, G. A.; Aaserud, D. J.; Fridriksson, E. K.; McLafferty, F. W. Top Down Versus Bottom Up Protein Characterization by Tandem High-Resolution Mass Spectrometry. J. Am. Chem. Soc. 1999, 121, 806-812.

5. Zubarev, R. A.; Mann, M. On the Proper Use of Mass Accuracy in Proteomics. Mol. Cell. Proteom. 2007, 6.3, 377-381.

6. Liu, T.; Belov, M. E.; Jaitly, N.; Qian, W.-J.; Smith, R. D. Accurate Mass Measurements in Proteomics. Chem. Rev. 2007, 107, 3621-3653.

7. Mann, M.; Kelleher, N. L. Precision Proteomics: The Case for High Resolution and High Mass Accuracy. Proc. Natl. Acad. Sci. U.S.A. 2008, 105, 18132-18138.

8. Zubarev, R. A.; Hakansson, P.; Sundqvist, B. Accuracy Requirements for Peptide Characterization by Monoisotopic Molecular Mass Measurements. Anal. Chem. 1996, 68, 4060-4063.

9. Spengler, B. De Novo Sequencing, Peptide Composition Analysis, and Composition-Based Sequencing: A New Strategy Employing Accurate Mass Determination by Fourier Transform Ion Cyclotron Resonance Mass Spectrometry. J. Am. Soc. Mass Spectrom. 2004, 15, 703-714.

10. Scheltema, R. A.; Kamleh, A.; Wildridge, D.; Ebikeme, C.; Watson, D. G. Barrett, M. R.; Jansen, R. C.; Breitling, R. Increasing the Mass Accuracy of High-Resolution LC-MS Data Using Background Ions-a Case Study on the LTQ-Orbitrap Proteomics 2008, 8, 4647-4656.

11. He, F.; Emmett, M. R.; Hakansson, K.; Hendrickson, C. L.; Marshall, A. G. Theoretical and Experimental Prospects for Protein Identification Based Solely on Accurate Mass Measurement. J. Proteom. Res. 2004, 3, 61-67.

12. Jensen, O. N.; Podtelejnikov, A.; Mann, M. Delayed Extraction Improves Specificity in Database Searches by Matrix-Assisted Laser Desorption/ Ionization Peptide Maps. Rapid Commun. Mass Spectrom. 1996, 10, 1371-1378.

13. Conrads, T. P.; Anderson, G. A.; Veenstra, T. D.; Pasa-Tolic, L.; Smith, R. D. Utility of Accurate Mass Tags for Proteome-Wide Protein Identification. Anal. Chem. 2000, 72, 3349-3354.

14. Makarov, A. Electrostatic Axially-Harmonic Orbital Trapping: A Novel High-Performance Technique of Mass Analysis. Anal. Chem. 2000, 72, 1156-1162.

15. Olsen, J. V.; de Godoy, L. M.; Li, G.; Macek, B.; Mortensen, P; Persch, R.; Makarov, A. A.; Lange, O.; Horning, S.; Mann, M. Parts Per Million Mass Accuracy on an Orbitrap Mass Spectrometer Via Lock-Mass Injection Into a C-Trap. Mol. Cell. Proteom. 2005, 4, 2010-2021.

16. Makarov, A.; Denisov, E.; Lange, O.; Horning, S., Dynamic Range of Mass Accuracy in LTQ Orbitrap Hybrid Mass Spectrometer. J. Am. Soc. Mass Spectrom. 2006, 17, 977-982.

17. Gorshkov, M. V.; Nikolaev, E. N. Optimal Cyclotron Radius FT-ICR Spectrometry for High Resolution. Int. J. Mass Spectrom. Ion Processes 1993, 125, 1-8.

18. Schweikhard, L.; Ziegler, J.; Bopp, H.; Lutzenkirchen, K. The Trapping Condition and a New Instability of the Ion Motion in the Ion-Cyclotron Resonance Trap. Int. J. Mass Spectrom. Ion Processes 1995, 141, 77-90.

19. Tolmachev, A. V.; Robinson, E. W.; Wu, S.; Kang, H.; Lourette, N. M. Paša-Tolic, L.; Smith, R. D. Trapped-Ion Cell With Improved DC Potential Harmonicity for FT-ICR MS. J. Am. Soc. Mass Spectrom. 2008, 19, 586-597.

20. Mitchell, D. W.; Rockwood, A. L.; Smith, R. D. Frequency-Shifts and Modulation Effects Due to Solenoidal Magnetic-Field Inhomogeneities 
in Ion-Cyclotron Mass-Spectrometry. Int. J. Mass Spectrom. Ion Processes 1995, 141, 101-116.

21. Jeffries, J. B.; Barlow, S. E.; Dunn, G. H. Theory of Space-Charge Shift of Ion-Cyclotron Resonance Frequencies. Int. J. Mass Spectrom. Ion Processes 1983, 54, 169-187.

22. Ledford, E. B.; Rempel, D. E.; Gross, M. L. Space-Charge Effects in Fourier-Transform Mass Spectrometry-Mass Calibration. Anal. Chem. 1984, 56, 2744-2748.

23. Chen, S. P.; Comisarow, M. B. Simple Physical Models for CoulombInduced Frequency Shifts and Coulomb-Induced Inhomogeneous Broadening for Like and Unlike Ions in Fourier-Transform Ion-Cyclotron Resonance Mass-Spectrometry. Rapid. Commun. Mass Spectrom. 1991, 5, $450-455$.

24. Gorshkov, M. V.; Nikolaev, E. N. Optimal Cyclotron Radius for HighResolution FT-ICR Spectrometry. Int. J. Mass Spectrom. Ion Processes 1993, 125, 1-8.

25. Gorshkov, M. V. Marshall, A. G. Nikolaev, E. N. Analysis and Elimination of Systematic Errors Originating From Coulomb Mutual Interaction and Image Charge in Fourier Transform Ion Cyclotron Resonance Precise Mass Difference Measurements. J. Am. Soc. Mass Spectrom. 1993, 4, 855-868.

26. Francl, T. J.; Sherman, M. G.; Hunter, R. L.; Locke, M. J.; Bowers, W. D.; McIver, R. T. Experimental Determination of the Effects of Space-
Charge on Ion-Cyclotron Resonance Frequencies. Int. J. Mass Spectrom. Ion Processes 1983, 54, 189-199.

27. Easterling, M. L.; Mize, T. H.; Amster, I. J. Routine Part-Per-Million Mass Accuracy for High-Mass Ions: Space-Charge Effects in MALDI FT-ICR. Anal. Chem. 1999, 71, 624-632.

28. Masselon, C.; Tolmachev, A. V.; Anderson, G. A.; Harkewicz, R.; Smith, R. D. Mass Measurement Errors Caused by "Local" Frequency Perturbations in FTICR Mass Spectrometry. J. Am. Soc. Mass Spectrom. 2002, 13, 99-106.

29. Muddiman, D. C.; Oberg, A. L. Statistical Evaluation of Internal and External Mass Calibration Laws Utilized in Fourier Transform Ion Cyclotron Resonance Mass Spectrometry. Anal. Chem. 2005, 77, $2406-$ 2414

30. Wong, R. L; Amster, I. J. Sub-Part-Per-Million Mass Accuracy by Using Stepwise-External Calibration in Fourier Transform Ion Cyclotron Resonance Mass Spectrometry. J. Am. Soc. Mass Spectrom. 2006, 17, 16811691.

31. Audi, G.; Wapstra, A. H.; Thibault, C. The AME2003 Atomic Mass Evaluation (II). Tables, Graphs, and References. Nucl. Phys. A 2003, 729, 337-676.

32. McAlister, G.; Phanstiel, D.; Coon, J. J. A Dual Reaction Cell, ETDEnabled Orbitrap Mass Spectrometer for Top-Down Proteomics. Proceedings of the 56th ASMS Conference on Mass Spectrometry and Allied Topics; Denver, CO, June, 2008. 